\title{
An MPC approach to individual pitch control of wind turbines using uncertain LIDAR measurements
}

Mirzaei, Mahmood; Soltani, Mohsen; Poulsen, Niels Kjølstad; Niemann, Hans Henrik

Published in:

2013 European Control Conference (ECC)

Publication date:

2013

Link back to DTU Orbit

Citation (APA):

Mirzaei, M., Soltani, M., Poulsen, N. K., \& Niemann, H. H. (2013). An MPC approach to individual pitch control of wind turbines using uncertain LIDAR measurements. In 2013 European Control Conference (ECC) (pp. 490495). IEEE.

\section{General rights}

Copyright and moral rights for the publications made accessible in the public portal are retained by the authors and/or other copyright owners and it is a condition of accessing publications that users recognise and abide by the legal requirements associated with these rights.

- Users may download and print one copy of any publication from the public portal for the purpose of private study or research.

- You may not further distribute the material or use it for any profit-making activity or commercial gain

- You may freely distribute the URL identifying the publication in the public portal 


\title{
An MPC approach to individual pitch control of wind turbines using uncertain LIDAR measurements
}

\author{
Mahmood Mirzaei, Mohsen Soltani, Niels K. Poulsen and Hans H. Niemann
}

\begin{abstract}
Spatial distribution of the wind field exerts unbalanced loads on wind turbine structures and it is shown these loads could be mitigated by controlling each blade's angle individually (individual pitch control). In this work the problem of individual pitch control of a variable-speed variablepitch wind turbine in the full load region is considered. Model predictive control (MPC) is used to solve the problem. A new approach is proposed to simplify the optimization problem of MPC. We linearize the obtained nonlinear model for different operating points which are determined by the effective wind speed on the rotor disc and take the wind speed as a scheduling variable. The wind speed is measurable ahead of the turbine using LIDARs, therefore the scheduling variable is known for the entire prediction horizon. We consider uncertainty in the wind propagation, which is the traveling time of wind from the LIDAR measurement point to the rotor. An algorithm based on wind speed estimation and measurements from the LIDAR is devised to find an estimate of the delay and compensate for it before it is used in the controller. Comparisons between the MPC with error compensation, without error compensation and a benchmark cyclic pitch PI controller are given. The results show that with appropriate signal processing techniques, LIDAR measurements improve the performance of the wind turbine controller.
\end{abstract}

\section{INTRODUCTION}

In recent decades, there has been increasing interest in green energies, of which wind energy is one of the most important. Horizontal axis wind turbines are the most common wind energy conversion systems (WECS) and are hoped to be able to compete with fossil fuel power plants on energy price in near future. However, this demands better technology to reduce the electricity production price. Control can play an essential part in this context. This is because, on the one hand improved control methods can decrease the cost of energy by keeping the turbine close to its maximum efficiency. On the other hand, they can reduce structural fatigue and increase the lifetime of the wind turbine. There are several methods of wind turbine control, ranging from classical control methods, which are the most commonly used methods in real applications [1], to advanced control methods, which have been the focus of research in the past

This work is supported by the Radar@Sea and the CASED Projects.

M. Mirzaei is with Department of Applied Mathematics and Computer Science, Technical University of Denmark, 2800 Kongens Lyngby, Denmark mmir@imm.dtu.dk

M. Soltani is with Department of Energy Technology, Aalborg University, 6700 Esbjerg, Denmark sms @et.aau.dk

N. K. Poulsen is with Department of Applied Mathematics and Computer Science, Technical University of Denmark, 2800 Kongens Lyngby, Denmark nkp@imm.dtu.dk

H. H. Niemann is with Department of Electrical Engineering, Technical University of Denmark, 2800 Kongens Lyngby, Denmark hhn@elektro.dtu.dk few years [2]. Gain scheduling [3], adaptive control [4], MIMO methods [5], nonlinear control [6], robust control [7], model predictive control [8], $\mu$-Synthesis design [9] and robust MPC [10] are just to mention a few. Advanced model-based control methods are thought to be the future of wind turbine control, as they can conveniently employ new generations of sensors on wind turbines (e.g. LIDAR [11]), new generation, of actuators (e.g. trailing edge flaps [12]) and they also treat the turbine as a MIMO system. Model predictive control (MPC) has proved to be an effective tool to deal with multivariable constrained control problems [13]. As wind turbines are MIMO systems [5] with constraints on inputs and outputs, using MPC is reasonable. MPC has been an active area of research and has been successfully applied on different applications in the last decades [14].

In this work, we extend the idea of linear MPC to formulate a tractable predictive control of the nonlinear system of wind turbines. To do so, we use future values of the effective wind speed that acts as a scheduling variable in the model. LIDAR measurements are used to calculate the effective wind speed ahead of wind turbines [11]. Several works have considered wind turbine control using LIDAR measurements [15], [16] and [17]. However there are a number of issues with LIDAR measurements that need to be considered. Uncertainty in the measurements is one of the important issues. Small errors in the calculations of the wind propagation time can severely degrade the performance of the controller.

The paper is organized as follows. In section II, modeling of the wind turbine is explained, the nonlinear model is derived and a linear model whose parameters vary as a function of effective wind speed is given. In section III, our proposed method for solving model predictive control of the system is presented. Then, the control design is explained, and control objectives are discussed. In section IV, uncertainty in the LIDAR measurements are explained, and a method is proposed to reduce this source of uncertainty. Finally, in section V, simulation results are given.

\section{MODELING}

\section{A. Nonlinear model}

The dominant dynamics of the wind turbine come from its flexible structure. Several degrees of freedom could be considered to model the flexible structure, but for control design a few important degrees of freedom are considered. In this work we consider three degrees of freedom, namely the rotational degree of freedom (DOF), the drivetrain torsion and the tower fore-aft displacement. Nonlinearity of the wind 
turbine model mostly comes from its aerodynamics. Blade element momentum (BEM) theory is used to numerically calculate aerodynamic torque and thrust on the wind turbine [18]. Having aerodynamic torque and modeling the drivetrain and the tower fore-aft degrees of freedom with simple massspring-damper, the whole system equation with 3 degrees of freedom becomes:

$$
\begin{aligned}
J_{r} \dot{\Omega}_{r} & =\mathcal{Q}_{r}-C_{d}\left(\Omega_{r}-\frac{\Omega_{g}}{N_{g}}\right)-K_{d} \psi \\
\left(N_{g} J_{g}\right) \dot{\Omega}_{g} & =C_{d}\left(\Omega_{r}-\frac{\Omega_{g}}{N_{g}}\right)+K_{d} \psi-N_{g} \mathcal{Q}_{g} \\
\dot{\psi} & =\Omega_{r}-\frac{\Omega_{g}}{N_{g}} \\
M \ddot{x}_{t} & =\mathcal{Q}_{t}-C_{t} \dot{x}_{t}-K_{t} x_{t} \\
\mathcal{P}_{e} & =\mathcal{Q}_{g} \Omega_{g}
\end{aligned}
$$

In which $\mathcal{Q}_{r}$ and $\mathcal{Q}_{t}$ are aerodynamic torque and thrust, $J_{r}$ and $J_{g}$ are rotor and generator moments of inertia, $\psi$ is the drivetrain torsion, $\mathcal{Q}_{g}$ and $\Omega_{g}$ are the generator torque and rotational speed, $N_{g}$ is the gearbox ration, $C_{d}$ and $K_{d}$ are the drivetrain damping and stiffness factors, respectively, lumped in the low speed side of the shaft. The tower mass, damping and stiffness factors are represented by $M, C_{t}$ and $K_{t}$, respectively, and $\mathcal{P}_{e}$ and $x_{t}$ are the generated electrical power and tower displacement, respectively. Values of the parameters can be found in [19].

\section{B. Linearized model}

To get a linear model of the system we need to linearize the model (1-5) around its operating points, which are determined by wind speed averaged on the rotor area. Wind speed changes along the blades and with the azimuth angle (angular position) of the rotor. This is because of wind shear, tower shadow and stochastic spatial distribution of the wind field. Therefore a single wind speed does not exist to be used and measured in order to find the operating point. We bypass this problem by defining a fictitious variable called effective wind speed $\left(\mathcal{V}_{e}\right)$, which shows the effect of wind on the rotor disc of the wind turbine. Using the linearized aerodynamic torque and thrust, state space matrices for the 3 DOFs linearized model become:

$$
\begin{aligned}
\dot{\omega}_{r} & =\frac{\alpha_{1}\left(v_{e}\right)-c}{J_{r}} \omega_{r}+\frac{c}{J_{r}} \omega_{g}-\frac{k}{J_{r}} \psi \\
& +\frac{\beta_{11}\left(v_{e}\right)}{J_{r}} \theta+\frac{\beta_{12}\left(v_{e}\right)}{J_{r}}\left(v_{e}-v_{t}\right) \\
\dot{\omega}_{g} & =\frac{c}{N_{g} J_{g}} \omega_{r}-\frac{c}{N_{g}^{2} J_{g}} \omega_{g}+\frac{k}{N_{g} J_{g}} \psi-\frac{Q_{g}}{J_{g}} \\
\dot{\psi} & =\omega_{r}-\frac{\omega_{g}}{N_{g}} \\
\dot{x}_{t} & =v_{t} \\
\dot{v}_{t} & =\frac{\alpha_{2}\left(v_{e}\right)}{M} \omega_{r}+\frac{\beta_{21}\left(v_{e}\right)}{M} \theta+\frac{\beta_{22}\left(v_{e}\right)}{M}\left(v_{e}-v_{t}\right) \\
& -\frac{C_{t}}{M} v_{t}-\frac{K_{t}}{M} x_{t} \\
P_{e} & =Q_{g_{0}} \omega_{g}+\omega_{g_{0}} Q_{g}
\end{aligned}
$$

In which the lower-case variables are deviations away from steady state of the upper-case variables given in the equations (1-5). Consequently, the parameters of the linearized model are functions of wind speed, which in our approach acts as a scheduling variable. A detailed description of the model and linearization is given in [9].

\section{Pitch actuator}

A second order model is used to model pitch actuator:

$$
\begin{aligned}
& \dot{\theta}_{1}=\theta_{2} \\
& \dot{\theta}_{2}=-2 \zeta_{\theta} \omega_{\theta} \theta_{2}-\omega_{\theta}^{2} \theta_{1}+\omega_{\theta}^{2} \theta_{i}
\end{aligned}
$$

In which $\theta_{i}$ is the input to the actuator, and $\omega_{\theta}$ and $\zeta_{\theta}$ are natural frequency and damping of the actuator, respectively.

\section{Dynamics of the blades}

Blade dynamics is simplified as a parameter varying second order dynamical system with two inputs, which are wind speed $\left(\tilde{v}_{i}\right)$ and pitch angle $\left(\tilde{\theta}_{i}\right)$, and one output, which is out-of-plane blade root bending moment $\left(\tilde{\mathcal{M}}_{i}\right)$. The model is considered to be:

$$
\tilde{\mathcal{M}}_{i}(s)=\mathcal{H}_{1, i}(\gamma, s) \tilde{\theta}_{i}(s)+\mathcal{H}_{2, i}(\gamma, s) \tilde{v}_{i}(s), \quad i=1,2,3
$$

This model shows the identified transfer functions for each of the blades, for which the state space matrices become:

$$
\begin{array}{ll}
A(\gamma)=\left(\begin{array}{cc}
0 & 1 \\
-\omega_{n}(\gamma)^{2} & -2 \zeta(\gamma) \omega_{n}(\gamma)
\end{array}\right) & C=\left(\begin{array}{cc}
1 & 0
\end{array}\right) \\
B(\gamma)=\left(\begin{array}{cc}
0 & 0 \\
b_{1}(\gamma) & b_{2}(\gamma)
\end{array}\right) & D=\left(\begin{array}{ll}
0 & 0
\end{array}\right)
\end{array}
$$

The argument $\gamma$ signifies that the linearized model depends on the operating point. Different wind speeds, which result in different operating points are used to identify the system. Prediction error [20] method is used on the above greybox model of the blade and parameters of the state space model are identified. It was observed that $\omega_{n}$ and $\zeta$ take almost constant values of $6(\mathrm{rad} / \mathrm{s})$ and $0.6(\mathrm{Ns} / \mathrm{m})$ respectively. Therefore, for the three blades, the dynamics of the whole system could be written as:

$$
\begin{array}{rlrl}
\mathcal{A} & =\bigoplus_{i=1}^{3} A & \mathcal{B}\left(\gamma_{1}, \gamma_{2}, \gamma_{3}\right) & =\bigoplus_{i=1}^{3} B\left(\gamma_{i}\right) \\
\mathcal{C} & =\bigoplus_{i=1}^{3} C & \mathcal{D} & =\bigoplus_{i=1}^{3} D
\end{array}
$$

In $\mathcal{B}\left(\gamma_{1}, \gamma_{2}, \gamma_{3}\right)$, the three variables $\gamma_{1}, \gamma_{2}$ and $\gamma_{3}$ are determined by the effective wind speed on the corresponding blade.

\section{Controller Design}

Wind turbine control is a challenging problem as the dynamics of the system changes based on wind speed which has a stochastic nature. In this paper, we use the wind speed as the scheduling variable. With the advances in the LIDAR technology [11] it is possible to measure wind speed ahead of the turbine and this enables us to have the scheduling 
variable of the plant for the entire prediction horizon. As it was mentioned in section II, wind turbines are nonlinear dynamical systems and if we use the nonlinear model directly in the MPC formulation, the optimization problem associated with the MPC becomes non-convex. In general, non-convex optimization problems are very complicated to solve and there is no guarantee that we could achieve a global optimum. One way to avoid complex and non-convex optimization problems is to linearize the system around an equilibrium point and use the obtained linearized model as an approximation of the nonlinear model. However, for wind turbines, assumption of the approximate linear model does not hold for long prediction horizons. This is because the operating point of the system changes as a function of wind speed which, as mentioned, has a stochastic nature.

\section{A. Problem formulation}

Having the LPV model of the system we proceed to compute state predictions using the approach proposed in [21]. In our method the predicted state is a function of the current state $x_{k}$, the control inputs $u_{n}$, as well as the scheduling variable $\Gamma_{n}=\left(\gamma_{k+1}, \gamma_{k+2}, \ldots \gamma_{k+n}\right)^{T}$ for $n=$ $1,2, \ldots, N-1$ and we assume that the scheduling variable is known for the entire prediction. By stacking the states, inputs and the affine term $\left(\lambda_{k}\right)$ of the state space equations, we can write down the stacked predicted state as:

$$
X=\Phi(\Gamma) x_{k}+\mathcal{H}_{u}(\Gamma) U+\Phi_{\lambda}(\Gamma) \Lambda
$$

After computing the state predictions as functions of control inputs, it is straightforward to write down the optimization problem similar to a linear MPC problem as a quadratic program, more details can be found in [21].

\section{B. Control objectives}

The most basic control objective of a wind turbine is to maximize captured power during the life time of the wind turbine that is to maximize captured power when wind speed is below its rated value. This is also called maximum power point tracking (MPPT). However when wind speed is above rated, control objective becomes regulation of the outputs around their rated values while trying to minimize dynamic loads on the structure. These objectives should be achieved against fluctuations in wind speed which acts as a disturbance to the system. In this work we have considered operation of the wind turbine in above rated (full load region). Therefore, we try to regulate rotational speed and generated power around their rated values and remove the effect of wind speed fluctuations.

\section{Implementation}

Two controllers are implemented in this work. One controller determines the collective pitch and generator reaction torque and regulates power and rotational speed. The second controller determines $\Delta \theta_{i}, i=1,2,3$ for fatigue load reduction by adjusting individual blade pitch based on the blade root bending moment measurements. The first controller takes advantage of having the wind speed for the entire prediction horizon, while the second controller uses only measurements from sensors at the blades root.

1) Collective pitch controller: The first controller uses the linearized model which was explained in section II-B augmented with a second order system modeling actuator dynamics. Measured outputs that are fed to this controller are:

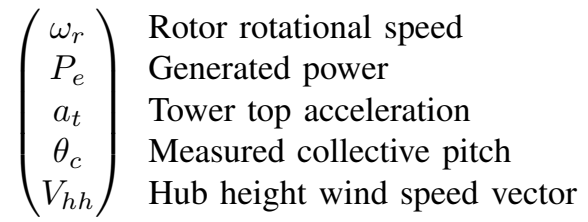

More details about this controller can be found in [21].

2) Individual pitch controller: The objective of this controller is to reduce fatigue loads on the blades by adjusting pitch angle of each blade based on blade root bending moment measurements. The method used here is similar to the one used in [22], except that we have used MPC instead of an LQG controller.

A multi-blade coordinate (MBC) transformation (also known as Coleman transformation) [23] is used to map fluctuations of the blade root bending moments $\left(\mathcal{M}_{i}, i=\right.$ $1,2,3)$ into a fixed coordinate system $\left(\mathcal{M}_{i}^{m b c}, i=1,2,3\right)$. Using the blades models derived in section II, an MPC is designed to reduce fluctuations on $\mathcal{M}_{i}^{m b c}, i=1,2,3$. The model predictive controller produces input signals which are then using inverse of MBC transformation transformed to pitch of the blades $\left(\Delta \theta_{i}, i=1,2,3\right)$.

\section{Benchmark controller}

The benchmark controller used in this work generates $\theta_{i}=\theta_{c}+\Delta \theta_{i}, i=1,2,3$, and consists of a collective pitch controller that generates $\theta_{c}$ and an individual controller that generates $\Delta \theta_{i}, i=1,2,3$. The collective pitch controller is based on the one found in [19]. The controller has a gainscheduled feedback from rotor speed to collective pitch angle and controls the generator torque to achieve constant power. The individual pitch control (IPC) system uses the flapwise blade root bending moments via the Coleman transform to determine cyclic behavior of the pitch angles. The cyclic pitch terms are then added to the collective pitch angle. Details regarding the tuning and implementation of the IPC can be found in [24].

\section{UNCERTAIN LIDAR MEASUREMENTS}

LIDAR measurements are used to have a preview of the wind speed [11], however these measurements are erroneous and uncertain. In this work, we have considered the uncertainties to be the measurement noise and uncertainty in the estimation of the wind propagation time. The propagation time is the time that it takes for the wind to travel from the LIDAR measurement points to the rotor disc. Lead or lag errors in the calculations of the propagation time severely reduce the performance of the controller. In order to bypass this problem, in this work, we have proposed a method to estimate these errors. To do so, we have designed an 


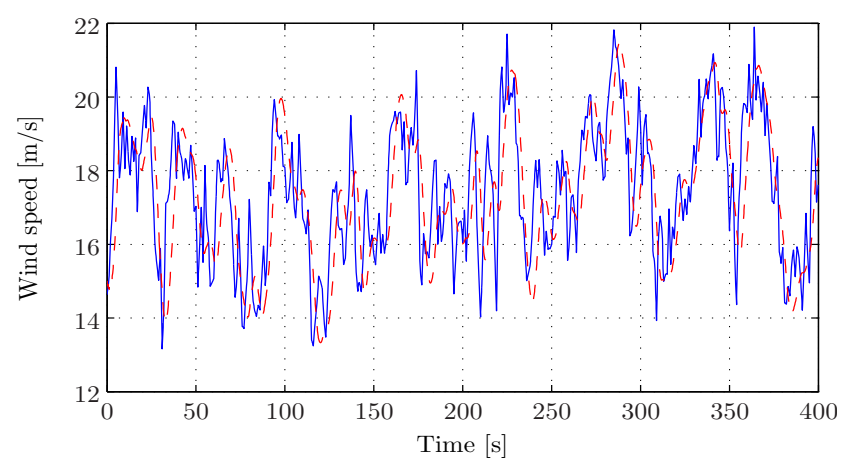

Fig. 1: Wind speed estimation (red-dashed line is the estimated wind speed and solid-blue line is the effective wind speed)

Extended Kalman filter which estimates the effective wind speed on the rotor plane. Then this estimate is compared against the filtered information that comes from the LIDAR measurements. Cross-covariance of the estimated wind speed and the LIDAR measurements are used to get an estimate of the delay between the two signals. Subsequently, the estimated delay is compensated for in LIDAR measurements and the resulting wind speed information is fed to the controller.

\section{A. Wind speed estimation}

Wind speed estimation is essential in our control algorithm. A one DOF model of the wind turbine, including only rotor rotational degree of freedom is used for wind speed estimation. This model is augmented with a linear model of the effective wind speed. The effective wind speed can be modeled as a complicated nonlinear stochastic process. However, for practical control purposes, it could be approximated by a linear model [25]. In this model, the wind has two elements, mean value term $\left(v_{m}\right)$ and turbulent term $\left(v_{t}\right)$. The mean wind speed varies relatively slowly and could be considered constant during one simulation. The turbulent term could be modeled by the following transfer function:

$$
v_{t}=\frac{k}{\left(p_{1} s+1\right)\left(p_{2} s+1\right)} e ; \quad e \in N(0,1)
$$

The parameters $p_{1}, p_{2}$ and $k$ which depend on the mean wind speed $v_{m}$ could be found by second order approximation of the wind power spectrum [25]. This state space model is augmented with the following model to be used in the extended Kalman filter:

$$
\begin{aligned}
\dot{\Omega} & =\frac{1}{J_{r}} \mathcal{Q}_{r}\left(\Omega, \Theta, \mathcal{V}_{e}\right)-\frac{1}{J_{r}} \mathcal{Q}_{g} \\
y & =\left(\begin{array}{ll}
\Omega & \mathcal{P}_{e}
\end{array}\right)^{T}
\end{aligned}
$$

Figure 1 shows wind speed and its estimate.

\section{B. Lead-lag error estimation and compensation}

For lead-lag error estimation, cross covariance of the estimated wind speed and measurements from the LIDAR for a window of size $m$-seconds is found. The result is

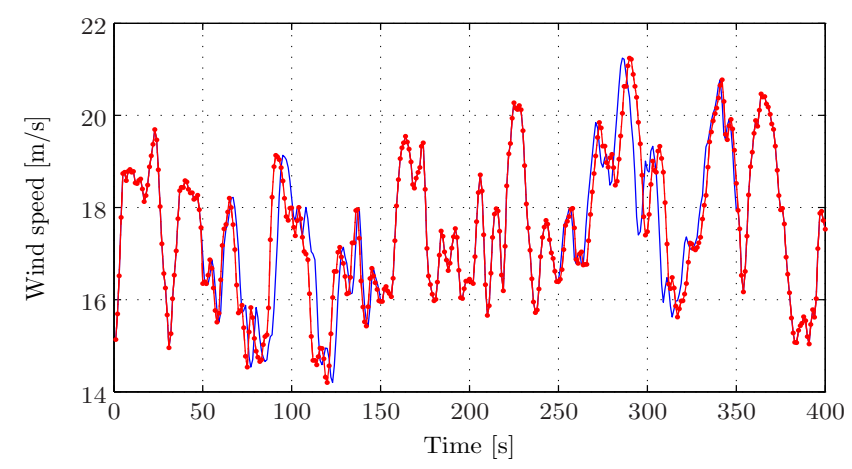

Fig. 2: Effective wind speed and LIDAR measurement with lead-lag errors (solid-blue is the effective wind speed, dottedred is the LIDAR measurement)

a sequence which has $(2 m-1)$ elements. By finding the maximum of the cross covariance, an estimate of the leadlag error can be found. The window size is important as it should be long enough to avoid erroneous results. The errors especially emerge when the window of effective wind speed signal has big autocorrelation values. By choosing a window with sufficiently large size this problem could be avoided. However, choosing a too big window size will result in slow delay detection which reduces performance of the controller. Cross covariance of the estimated wind speed and LIDAR measurements, can be found using the following formula:

$$
\phi_{\hat{v} v}(t)=E\left\{\left(\hat{v}_{n+t}-\mu_{\hat{v}}\right)\left(v_{n}-\mu_{v}\right)^{T}\right\}
$$

in which $\hat{v}$ is the estimated wind speed and $v$ is the LIDAR measurements. Having the sequence of $\phi_{\hat{v} v}(t)$, one can calculate lead-lag error by the following formula:

$$
t_{e}=\arg \max _{t} \phi_{\hat{v} v}(t)
$$

in which $t_{e}=t_{\text {measurment }}-t_{\text {actual wind speed. }} t_{e}$ is then passed through a low pass filter to remove fluctuations due to numerical errors and possible autocorrelations. Then it is used to shift LIDAR measurements. Afterwards the shifted signal is used in the controller. Figure 2 shows a comparison of the effective wind speed and the wind speed measured by LIDAR. There is a 4 seconds lead error at time $100 \mathrm{~s}$ (in which the measurement is lead) and then at time $300 \mathrm{~s}$ the same amount of lag error. Figure 3 shows a comparison between the introduced delay in the measurements and its estimation. The lead-lag error estimation is delayed, however it follows the shape of the actual delay. In the worst cases, when the LIDAR measurements does not give a good correlation with the wind speed estimation on the turbine, the measurements could be discarded and the turbine can operate without LIDAR measurements.

\section{Simulations}

In this section, simulation results for the obtained controllers are presented. The controllers are implemented in MATLAB and tested on a high fidelity wind turbine simulation software FAST [26] using the model of the reference wind turbine [19]. The results of the proposed approach 


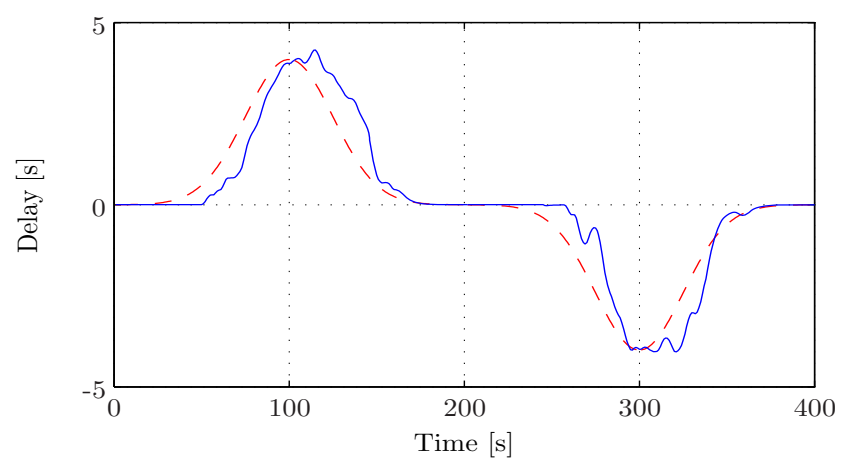

Fig. 3: Comparison of introduced delay and its estimation (solid-blue is the estimated delay, dashed-red is the introduced delay)

TABLE I: Performance comparison (SD stands for standard deviation)

\begin{tabular}{lccc} 
Parameters & $\begin{array}{c}\text { MPC+LIDAR+ } \\
\text { Compensation }\end{array}$ & $\begin{array}{c}\text { MPC+ } \\
\text { LIDAR }\end{array}$ & $\begin{array}{c}\text { Cyclic } \\
\text { pitch PI }\end{array}$ \\
\hline SD of $\omega_{r}(\mathrm{RPM})$ & 0.398 & 1.22 & 0.703 \\
SD of $P_{e}$ (k Watts) & 76.5 & 215 & 89.0 \\
Pitch travel (degrees) & 525.4 & 562.5 & 484.5 \\
SD of shaft moment $(\mathrm{k}$ N.M.) & 1.21 & 3.78 & 2.30 \\
SD of tower acc. $\left(\mathrm{m} / \mathrm{s}^{2}\right)$ & 0.229 & 0.284 & 0.254
\end{tabular}

with lead-lag error estimation are compared against two controllers. One of them is an MPC with the same tunings and with the same LIDAR measurements but without error compensation. The other controller is a benchmark cyclic pitch PI controller [22]. Simulations are done using turbulent wind speed, with Kaimal model [27]. And TurbSim [28] is used to generate the wind profile. In order to stay in the full load region, a realization of turbulent wind speed is used from category $C$ of the turbulence categories of the IEC 61400-1 [27] with the mean wind speed of $18 \mathrm{~m} / \mathrm{s}$. Wind shear is included with 0.2 as the value for the power-law exponent. Control inputs are individual pitch of the blades $\theta_{i}, i=1,2,3$ and generator reaction torque $Q_{g}$. System outputs are rotor rotational speed $\omega_{r}$, electrical power $P_{e}$, tower fore-aft acceleration $\ddot{x}_{t}$ and out-of-plane blade root bending moments which are plotted in figures 4-8. Table I shows a comparison of the results between the proposed approach with lead-lag error estimation, the linear MPC with LIDAR measurements and without compensation and finally the cyclic pitch PI controller. For comparisons, we have used pitch travel $\left(\int|d \theta / d t| d t\right)$ to take into account an approximation of the damage on the pitch actuator. Standard deviations (SD) of the rotational speed and generated power are also compared. To compare fluctuations of the blade root bending moments, we have used power spectral density (PSD) of the signals and compared them in figure 9. As it can be seen in the table I and figures 4-9, the proposed approach gives better regulation on rotational speed and generated power (smaller standard deviations) and less fluctuations on the tower fore-aft and blade root bending moments, while maintaining less drivetrain torsion. However the pitch travel is slightly more than the cyclic PI controller which could be

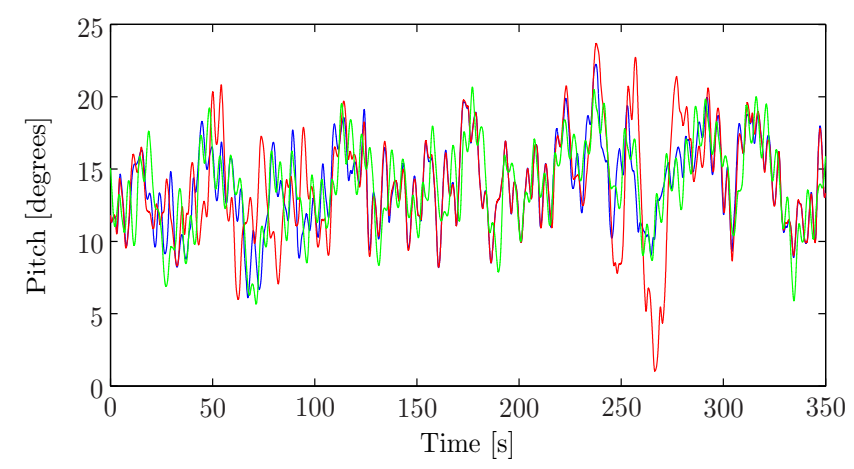

Fig. 4: Blade-pitch (blue line is MPC with LIDAR and delay compensation, red line is MPC with LIDAR without delay compensation and green line is PI-cyclic pitch)

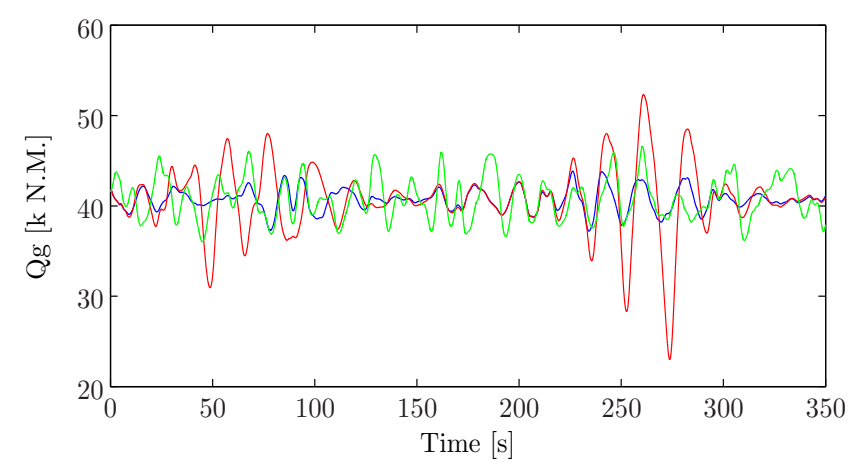

Fig. 5: Generator-torque (blue line is MPC with LIDAR and delay compensation, red line is MPC with LIDAR without delay compensation and green line is PI-cyclic pitch)

reduced by tuning.

\section{CONCLUSIONS}

LIDAR measurements improve performance of wind turbines. However, errors in the calculation of the wind propagation time severely degrade the performance of the controller. In this work, we have shown that using appropriate signal processing techniques, these errors can be removed form the measurements and even in the worst cases, when LIDAR measurements are not reliable, the turbine can operate without using the data from the LIDAR.

\section{REFERENCES}

[1] W.E. Leithead and Bill Connor. Control of variable speed wind turbines: Design task. Int J Control, 73(13):1189-1212, 2000.

[2] J.H. Laks, L.Y. Pao, and A.D. Wright. Control of wind turbines: Past, present, and future. Proceedings of the American Control Conference, pages 2096-2103, 2009.

[3] Fernando D. Bianchi, Hernan De Battista, and Ricardo J. Mantz. Wind Turbine Control Systems: Principles, Modelling and Gain Scheduling Design. Springer, 2006.

[4] Johnson and Fingersh. Adaptive pitch control of variable-speed wind turbines. J. Sol. Energy Eng. (USA), 130(3):031012-1-7, 2008.

[5] M. Geyler and P. Caselitz. Robust multivariable pitch control design for load reduction on large wind turbines. Journal of solar energy engineering, 130(3):-, 2008.

[6] Sven Creutz Thomsen. Nonlinear control of a wind turbine. Master's thesis, Technical University of Denmark, Informatics and Mathematical Modelling, Lyngby, Denmark, 2006. 


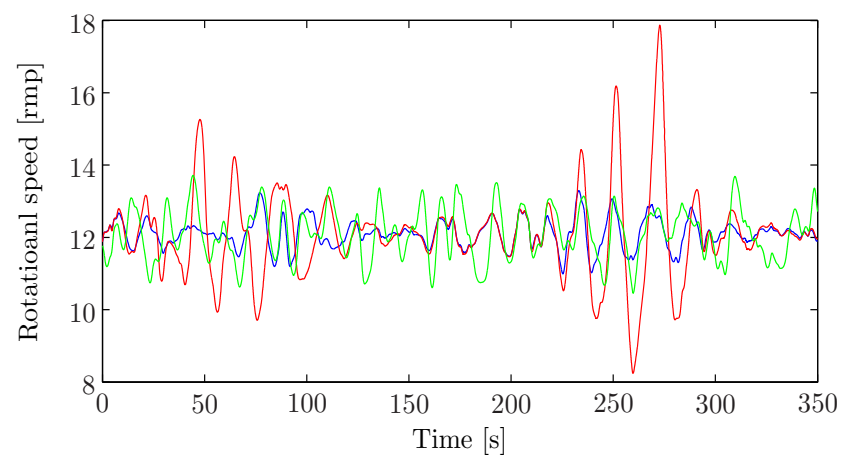

Fig. 6: Rotor rotational speed (blue line is MPC with LIDAR and delay compensation, red line is MPC with LIDAR without delay compensation and green line is PI-cyclic pitch)

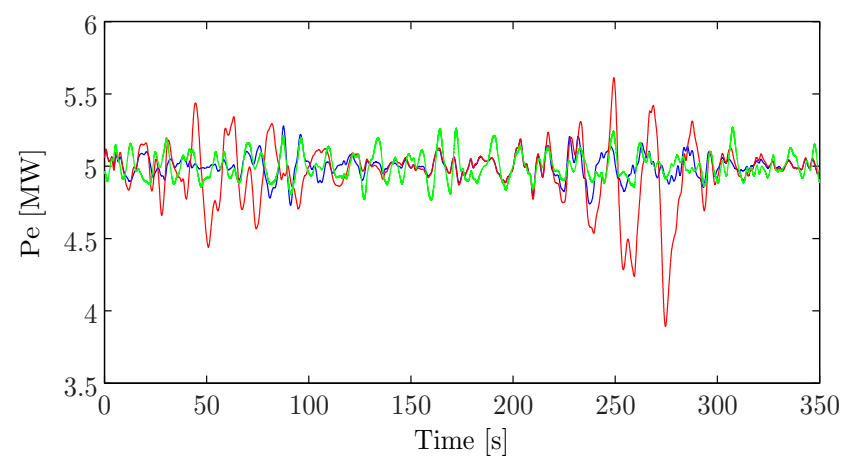

Fig. 7: Electrical power (blue line is MPC with LIDAR and delay compensation, red line is MPC with LIDAR without delay compensation and green line is PI-cyclic pitch)

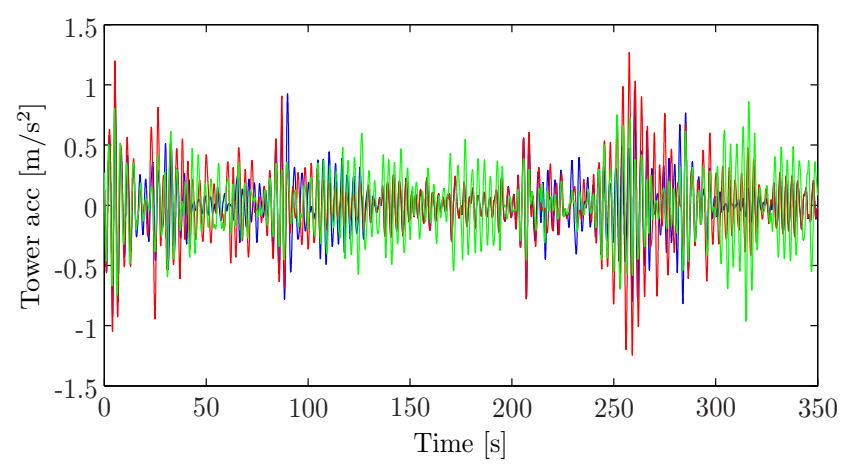

Fig. 8: Tower top acceleration (blue line is MPC with LIDAR and delay compensation, red line is MPC with LIDAR without delay compensation and green line is PI-cyclic pitch)

[7] Kasper Zinck Østergaard. Robust, Gain-Scheduled Control of Wind Turbines. PhD thesis, Automation and Control Department of Electronic Systems, Aalborg University, 2008.

[8] Lars Christian Henriksen. Model predictive control of a wind turbine. Master's thesis, Technical University of Denmark, Informatics and Mathematical Modelling, 2007.

[9] Mahmood Mirzaei, Hans Henrik Niemann, and Niels Kjølstad Poulsen. A $\mu$-synthesis approach to robust control of a wind turbine. In the 50th IEEE Conference on Decision and Control and European Control Conference, pages 645-650, Orlando, Florida, United States, 2011.

[10] Mahmood Mirzaei, Niels Kjølstad Poulsen, and Hans Henrik Niemann. Robust model predictive control of a wind turbine. In American Control Conference, Montral, Canada, 2012.

[11] M. Harris, M. Hand, and A. Wright. LIDAR for turbine control.

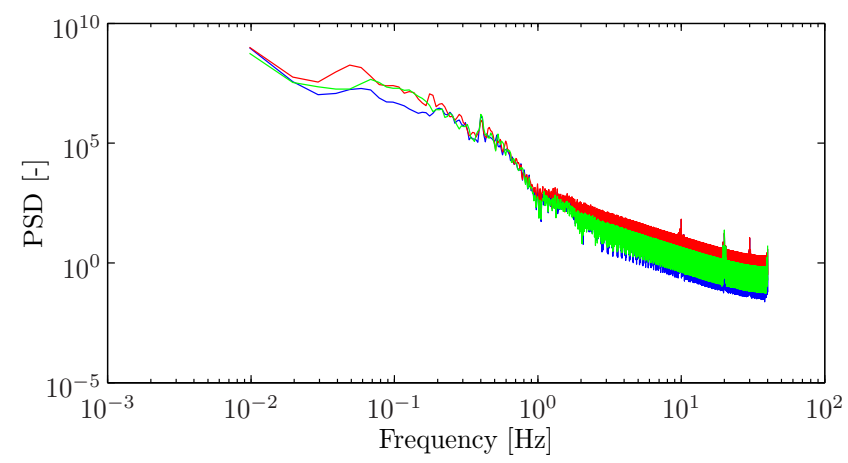

Fig. 9: Power spectral density of the out-of-plane blade root bending moments (blue line is MPC with LIDAR and delay compensation, red line is MPC with LIDAR without delay compensation and green line is PI-cyclic pitch)

Technical report, National Renewable Energy Laboratory, 2006.

[12] Peter Bjørn Andersen. Advanced Load Alleviation for Wind Turbines using Adaptive Trailing Edge Flaps: Sensoring and Control. $\mathrm{PhD}$ thesis, Technical University of Denmark, Denmark, 2010.

[13] J.M. Maciejowski. Predictive control with constraints. Pearson Education Lim., Essex, 2002. 1-331 s pp.

[14] S. Joe Qin and Thomas A. Badgwell. An overview of industrial model predictive control technology. pages -, 1996.

[15] M. Soltani, R. Wisniewski, P. Brath, and S. Boyd. Load reduction of wind turbines using receding horizon control. The proceedings of the IEEE Conference on Control Applications, pages 852-857, 2011.

[16] Jason Laks, Lucy Y. Pao, Eric Simley, Alan Wright, Neil Kelley, and Bonnie Jonkman. Model predictive control using preview measurements from lidar. In 49th AIAA Aerospace Sciences Meeting including the New Horizons Forum and Aerospace Exposition, Orlando, Florida, January 2011.

[17] Mahmood Mirzaei, Niels Kjølstad Poulsen, and Hans Henrik Niemann. Individual pitch control using lidar measurements. In IEEE Multiconference on Systems and Control, Dubrovnik, Croatia, 2012.

[18] Martin O. L. Hansen. Aerodynamics of Wind Turbines. Earthscan, 2008.

[19] J. Jonkman, S. Butterfield, W. Musial, and G. Scott. Definition of a $5 \mathrm{MW}$ reference wind turbine for offshore system development. Technical report, National Renewable Energy Laboratory,, 1617 Cole Boulevard, Golden, Colorado 80401-3393 303-275-3000, 2009.

[20] L. Ljung. System identification / Theory for the user. Prentice Hall, Upper Saddle River, N.J., 1999. 1-609 s pp.

[21] Mahmood Mirzaei, Niels Kjølstad Poulsen, and Hans Henrik Niemann. Model predictive control of a nonlinear system with known scheduling variable. Proceedings of the 17th Nordic Process Control Workshop, pages 163-168, 2012.

[22] E. A. Bossanyi. Individual blade pitch control for load reduction. Wind Energy, 6:119128, 2002.

[23] Robert P Coleman and Arnold M Feingold. Theory of self-excited mechanical oscillations of helicopter rotors with hinged blades. CASI, pages -, 1961.

[24] Edited by Morten Hartvig Hansen and Frederik Zahle. Aeroelastic optimization of mw wind turbines. Technical Report Ris $\varnothing-\mathrm{R}-1803$, Risø National Laboratory for Sustainable Energy, Technical University of Denmark, December 2011.

[25] Torben Knudsen, Thomas Bak, and Mohsen Soltani. Prediction models for wind speed at turbine locations in a wind farm. Wind Energ., 14 (7):877-894, 2011.

[26] Jason M. Jonkman and Marshall L. Buhl Jr. Fast user's guide. Technical Report NREL/EL-500-38230, National Renewable Energy Laboratory, Golden, CO, August 2005.

[27] IEC 61400-1 wind turbines-part 1: Design requirements., August 2005.

[28] B.J. Jonkman. Turbsim user's guide: Version 1.50. Technical report, National Renewable Energy Laboratory, 1617 Cole Boulevard, Golden, Colorado 80401-3393 303-275-3000, 2009. 